\begin{tabular}{|c|c|}
\hline 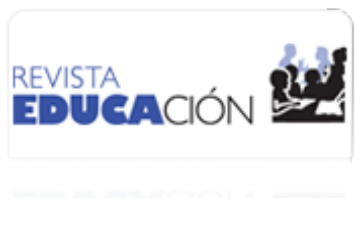 & $\begin{array}{l}\text { Revista Educación } \\
\text { ISSN: 0379-7082 } \\
\text { ISSN: } 2215-2644 \\
\text { revedu@gmail.com } \\
\text { Universidad de Costa Rica } \\
\text { Costa Rica }\end{array}$ \\
\hline
\end{tabular}

\title{
El sistema de promoción en la educación media: Criterio de la comunidad educativa
}

\author{
Chaves Salas, Ana Lupita; Castro Bonilla, Julieta; García Fallas, Jacqueline \\ El sistema de promoción en la educación media: Criterio de la comunidad educativa \\ Revista Educación, vol. 41, núm. 2, 2017 \\ Universidad de Costa Rica, Costa Rica \\ Disponible en: http://www.redalyc.org/articulo.oa?id=44051357005 \\ DOI: http://dx.doi.org/10.15517/revedu.v41i2.23653
}

Esta obra está bajo una Licencia Creative Commons Atribución-NoComercial-SinDerivar 3.0 Internacional. 


\section{El sistema de promoción en la educación media: Criterio de la comunidad educativa}

\section{The promotion system at high school: criteria of the educational community}

Ana Lupita Chaves Salas [1]

Universidad de Costa Rica, Costa Rica

lupitachaves@gmail.com

Julieta Castro Bonilla [2]

Universidad de Costa Rica, Costa Rica

julietacastrob@gmail.com

Jacqueline García Fallas [3]

Universidad de Costa Rica, Costa Rica

jgarciafallas@gmail.com
DOI: http://dx.doi.org/10.15517/revedu.v41i2.23653

Redalyc: http://www.redalyc.org/articulo.oa?id=44051357005

\section{Resumen:}

Este artículo tiene como propósito dar a conocer los resultados de un proyecto de investigación que se llevó a cabo en el Instituto de Investigación en Educación (INIE), Facultad de Educación de la Universidad de Costa Rica (UCR), cuyo objetivo general fue analizar el criterio que tiene la comunidad educativa sobre la Reforma de las Normas reguladoras de la promoción y repitencia del Sistema Educativo Costarricense. Para lograrlo, se realizó una investigación cualtitativa descriptiva de tipo exploratorio, en la que participó la comunidad educativa de dos centros de educación secundaria públicos, además, autoridades del Ministerio de Educación Pública (MEP), docentes de universidades y de la Asociación Nacional de Educadores (ANDE). La investigación inicia con un acercamiento a los antecedentes de esta Reforma y, posteriormente, se continúa con una revisión bibliográfica sobre el tema. El estudio evidencia que la comunidad educativa manifiesta no estar de acuerdo con este sistema de promoción de la educación media en Costa Rica y ofrece sugerencias para mejorarlo.

PALABRAS ClAVE: Educación secundaria, evaluación de los aprendizajes, promoción y repitencia, reforma educativa, Ministerio de Educación Pública, Costa Rica.

\section{Abstract:}

The purpose of this article is to reveal the research project results that took place in the Educational Research Institute (INIE), Education School, at the University of Costa Rica (UCR). The research main objective was to analyze the educational

\section{NotAS DE AUTOR}

[1] Doctora en Educación. Magister en Educación de Adultos. Bachiller y Licenciada en Educación Preescolar. Actualmente es representante de la Universidad de Costa Rica en el Consejo Superior de Educación. Fue decana de Facultad de Educación de la Universidad de Costa Rica y directora del Programa de Posgrado Académico en Educación. Es investigadora del Instituto de Investigación en Educación de la UCR y fue su directora del 2001 al 2008. Docente de la carrera de Bachillerato y Licenciatura en Educación Preescolar de la Escuela de Formación Docente. Directora de tesis de grado y posgrado. Participa como ponente en congresos nacionales e internacionales. Es miembro del Consejo Editorial de la Revista en español de la International Reading Association y de la Revista de Educación de la Universidad Central de Venezuela. Es autora de libros, capítulos de libros y numerosos artículos en revistas nacionales y de otros países sobre una diversidad de temas: educación preescolar, procesos iniciales de lectoescritura, investigación-acción en el aula, formación docente, escuelas unidocentes, docentes destacados, evaluación en secundaria, entre otros. Coordina proyectos de extensión dirigidos a docentes en zonas urbanas y rurales.

[2] Doctora en Educación de la Universidad de Costa Rica. Labora como investigadora en el Instituto de Investigación en Educación y como docente en la Escuela de Filosofía de la Universidad de Costa Rica. Actualmente es la directora de este instituto. Ha participado como ponente en congresos nacionales e internacionales. Entre sus publicaciones recientes se destacan: "Uso de un portafolio virtual para incorporar la dimensión ambiental: una experiencia de investigación-acción en el aula”; Construcción de una comunidad virtual de aprendizaje ambiental (CVAA): espacio interactivo para ambientalizar el quehacer universitario estatal de Costa Rica; Educación ambiental: Una estrategia para ambientalizar el currículo universitario;. Percepción sobre la dimensión ambiental en el ámbito personal y laboral: aplicación y valoración de un pretest., entre otras.

[3] Fue profesora de segunda enseñanza con énfasis en artes plásticas, de la Facultad de Educación, Universidad de Costa Rica. Actualmente es investigadora del Instituto de Investigación en Educación de dicha Facultad. Tiene los títulos de Bachiller en Artes Plásticas, por Facultad de Bellas Artes, Bachiller en Ciencias de la Educación con énfasis en enseñanza media, por la Facultad de Educación, Licenciada en Bellas Artes con especialidad en Pintura, por la Facultad de Bellas Artes, todos estos grados fueron obtenidos en la Universidad de Costa Rica, y Master of Science in Education de la State University of New York, College at Buffalo, New York, E.U.A. Es autora de libros, capítulos de libros y numerosos artículos en revistas nacionales sobre diferentes temas de la educación y de su especialidad, también coordina proyectos de extensión dirigidos a docentes en zonas urbanas y rurales. 
community's criterion related to "Reform of regulatory rules of promotion and repetition in the Costa Rican educational system". Furthermore, this research involved a change in the promotion system of the secondary students in curriculum, teaching, and administrative aspects. Moreover, the objective was achieved by using a descriptive exploratory qualitative research methodology, in which the educational community of two public secondary schools, Ministry of Education authorities (MEP), university professors, and the National Association of Teachers (ANDE) participates. This research begins with the reform's antecedents and then a literature review about the topic. Therefore, there is evidence in this study related to the educational community's disagreement about this reform and they propose suggestions in order to improve the high school Promotion System in Costa Rica. KEYWORDS: high school, learning assessment, promotion and repetition, educational reform, Ministry of Public Education, Costa Rica.

\section{INTRODUCCIÓN}

Este artículo presenta los resultados de la investigación "El sistema de promoción en la educación media: Criterio de la comunidad educativa", la cual se desarrolla en dos instituciones públicas de educación secundaria. Para llevarla a cabo se aplican cuestionarios y entrevistas al personal administrativo, docentes, estudiantes y sus respectivas familias. Asimismo, se toman en cuenta las opiniones de autoridades del Ministerio de Educación Pública (MEP), de la Asociación Nacional de Educadores y personal docente universitario mediante entrevistas y grupos focales. Esas personas emiten su criterio con respecto a la situación académica de la educación media y su percepción sobre esta Reforma, para generar aprendizajes a partir del análisis de las opiniones generadas. Cabe señalar que la "Reforma de las Normas reguladoras de la promoción y repitencia del Sistema Educativo Costarricense” se implementó en el año 2009 en el sistema educativo costarricense.

Para la ejecución de este proceso, también se valora la experiencia de las investigadoras en diversos contextos educativos, la documentación relativa a la Reforma, así como investigaciones y bibliografía sobre el tema.

\section{ANTECEDENTES}

\subsection{Plan Nacional de Desarrollo Educativo (1971)}

Para efectos de la presente investigación, se estudia el Plan Nacional de Desarrollo Educativo (Ministerio de Educación Pública, MEP,1971), el cual se promulga siendo Ministro de Educación el señor Uladislao Gámez Solano. Para la organización y planificación de esa Reforma Educativa se solicita el asesoramiento y criterio a diferentes instituciones y entidades: la UNESCO y la Fundación Friedrich, la cual ofrece apoyo por medio del Centro de Estudios Democráticos de América Latina (CEDAL), en Costa Rica. Tomando en cuenta las respectivas recomendaciones, se inicia un trabajo a lo interno del Ministerio de Educación Pública. Se resuelven algunos problemas administrativos en el Departamento de Personal, se crea el Departamento de Currículum, se instala la Oficina de Planeamiento Integral, como oficina sectorial de OFIPLAN, se constituyen comisiones integradas por docentes que laboran para el sistema educativo costarricense con formación en educación preescolar, primaria, enseñanza media, así como docentes que laboran para la Universidad de Costa Rica; con el propósito de analizar las sugerencias brindadas por esas instituciones y entidades.

También se contrata un estudio relacionado con la realidad educativa nacional, y se publica una síntesis con el título de Diagnóstico, y como consecuencia de ello se abandona el concepto de Reforma y se propone una labor de mayor profundidad al que se le denomina "Plan Nacional de Desarrollo Educativo". El trabajo realizado y sus alcances, se resumen en tres folletos titulados: Diagnóstico, Los grandes objetivos y La 
programación. Finalmente se publica la obra final, la cual contó con la participación de autoridades y especialistas en los temas de interés.

Como resultado del diagnóstico de la educación nacional, se encontró que había un porcentaje bastante elevado de escolares que repiten principalmente el "primer grado", situación que incidía de manera directa en la deserción. En el caso de la enseñanza media, el texto señala que "el problema principal es la deserción, especialmente en los dos primeros años, aunque luego también existe una tasa no despreciable de repetición” (Ministerio de Educación Pública,1971, p. 5).

Y continúa señalando que, de acuerdo con el estudio, “...estos fenómenos elevan extraordinariamente el costo para el Estado de cada graduado [también] ...reflejan... el bajo provecho que, para la sociedad, ofrece el actual sistema de educación" (Ministerio de Educación Pública, 1971, p. 7). En parte, esos resultados son atribuidos a una serie de condiciones, referentes al contexto socioeconómico y a las realidades del proceso educativo: contexto escolar, desarrollo de planes y programas, formación docente, características del estudiantado, sin obviar el sistema de evaluación y promoción.

\section{La evaluación y la promoción en el Plan Nacional de Desarrollo Educativo}

El concepto de evaluación que fundamenta el Plan Nacional de Desarrollo Educativo es considerado "un instrumento para ayudar a los alumnos, en forma constante y desde el primer día de clases: es una información continua sobre los resultados del aprendizaje, a fin de corregir a tiempo... lo que haya de defectuoso en aquél" (Ministerio de Educación Pública, 1971, p. 60). De esta manera, se gesta una evaluación continua y permanente, la cual se convierte en una herramienta que le facilita al personal docente registrar los resultados de sus prácticas profesionales para mejorarlas; asimismo, el alumnado podía apreciar en él mismo, los resultados de su proceso de aprendizaje y verificar si requería o no de una atención personalizada por parte del equipo docente.

El planteamiento evaluativo que emana de dicho Plan se considera continuo y formativo, ya que le permite al personal docente recuperar a sus estudiantes en el momento en que estos grupos lo requerían y bajo la tutela docente asignada, y de esta manera se podía rescatar su interés y motivación para avanzar en sus estudios. En este contexto, el Plan consideraba el espacio de tres años (I, II y III ciclos) para que la persona pudiera recuperarse, tanto individual como grupalmente. Es decir, si al final del I y II ciclo de primaria y III de secundaria, se presentan deficiencias en el dominio de algún contenido, "existe la posibilidad de un semestre o un año completo de recuperación, la cual se efectuará sobre aquellos puntos en que se hayan manifestado las deficiencias, sin obligarse al alumno a repetir lo que ya dominó" (Ministerio de Educación Pública 1971, p. 62). De esta manera, se pasa de un criterio de evaluación por ciclo y se elimina el que se tenía por grado.

El Reglamento General de Evaluación y Normas de Promoción, del Plan Nacional de Desarrollo Educativo, promovía una adecuación metodológica individualizada y grupal que favorecía la recuperación. A partir de esa adecuación, cada estudiante podía continuar su ciclo lectivo con normalidad (recordando que este está compuesto por tres años), pero debía asistir a lecciones especiales de "recuperación", cuando el profesorado u otra persona lo podía atender para apoyarlo únicamente en los aspectos curriculares que presentara carencias; concluido este proceso, tenía la oportunidad de presentar la prueba respectiva durante los meses de febrero, mayo o setiembre. Así, podía evidenciar que tenía los conocimientos necesarios, sin la necesidad de volver a demostrar o comprobar los conocimientos que ya había adquirido. El estudiantado matriculaba el curso lectivo completo y arrastraba las materias que había reprobado del año anterior.

Después de derogarse ese Plan a finales de los años setenta, se continuó con la normativa anterior, que estuvo vigente hasta el año 2008. Esa normativa estipulaba lo siguiente:

- El curso lectivo se reprueba con la pérdida de cuatro asignaturas, se permite presentar pruebas de ampliación en tres asignaturas. 
- Se reprueba una asignatura, si se obtiene una calificación inferior a sesenta en el tercer trimestre.

- Una calificación en disciplina menor a 60 implica la pérdida del año lectivo.

Esta normativa cambia al aprobar el Consejo Superior de Educación en el 2008 la Reforma de las Normas reguladoras de la promoción y repitencia del Sistema Educativo Costarricense que se implementan a partir del 2009 (Consejo Superior de Educación, 2008). Seguidamente se da a conocer el contenido de esta Reforma.

\subsection{Reforma de las Normas reguladoras de la promoción y repitencia del Sistema Educativo Costarricense (2009)}

La Reforma de las normas para la promoción y repitencia en educación secundaria tiene como uno de sus propósitos retener a la población estudiantil en la institución escolar puesto "...que la exclusión del Sistema educativo se mantiene como un problema de primer orden, especialmente en la enseñanza secundaria" (CONARE, 2008, p. 52).

De tal manera que esta Reforma implementa las siguientes normas de promoción:

- Se autoriza presentar prueba de ampliación hasta en cuatro asignaturas reprobadas, es decir, se aumenta una más.

- El estudiantado repite solo las asignaturas que reprueba con la posibilidad de adelantar otras de niveles superiores.

- Los cursos se aprueban con su nota ponderada anual (tercer trimestre no aplaza).

- La nota de conducta no afecta la evaluación de las asignaturas.

La Reforma que se implementa a partir del 2009 en las instituciones de educación secundaria (MEP, 2008), recibe críticas de la comunidad educativa, entre ellas: afecta la administración curricular en el centro de enseñanza, los procesos de enseñanza y aprendizaje en las aulas, así como la identificación y pertenencia del estudiantado con su grupo. Ante las críticas, el ministro de Educación Pública, señor Garnier Rímolo, indica que hay una alta relación entre repitencia y deserción; y la Reforma lo que pretende es “...atacar algunas de las que consideramos causas artificiales del fracaso escolar y de una repitencia injustificada, innecesaria y de severas consecuencias en términos de deserción y de la baja cobertura de la educación secundaria" (MEP, 2011, p. 2).

Para detener la deserción estudiantil, el MEP justifica la puesta en marcha de las normas de promoción señaladas e implementa el programa "Avancemos", el cual asigna recursos económicos a familias que los requieran para que sus hijas e hijos permanezcan en el sistema educativo.

Esas normas de promoción son valoradas como positivas por las autoridades del MEP durante el periodo 2009-2010; no obstante, se identifican algunas situaciones problemáticas respecto a su implementación:

Entre los principales problemas identificados destacan las complicaciones administrativas provocadas por la Reforma y la carencia de recursos -humanos y tecnológicos- para enfrentarlas de la mejor manera; también se argumenta que los estudiantes de secundaria no tienen aun la madurez necesaria para este tipo de sistema y que, en consecuencia, al quedarles "cajones" o ratos libres, se pierde mucho tiempo o faltan a clases, aumentando el ausentismo; otro problema que se ha identificado es que, al encontrarse las y los estudiantes en dos o tres niveles o en distintos grupos, puede perderse el sentido de pertenencia a "su grupo". Finalmente, algunos han argumentado que, a pesar de la reforma, los estudiantes no recuperan las asignaturas perdidas y nunca logran ponerse a nivel con su grupo original. (MEP, 2011, p. 3)

A partir de los problemas señalados por el MEP y otros actores sociales, el equipo de investigadoras se da a la tarea de indagar las experiencias -negativas y positivas- que han generado esos lineamientos en personal responsable e involucrado directo de su puesta en práctica en la institución escolar: cuerpo administrativo, docente, estudiantil y familiar, así como generar lecciones a partir del análisis de las opiniones generadas por las autoridades del MEP y la ANDE. 


\section{Marco teórico}

El marco teórico de la presente investigación corresponde a un proceso de construcción de las investigadoras, por medio del cual se indagan los conceptos afines al tema de la investigación para fundamentarla. Los conceptos que apoyan este proceso permiten la comprensión de las normas de promoción en la enseñanza secundaria.

\subsection{La educación secundaria y diversificada}

La educación secundaria comprende el III ciclo de la educación general básica que abarca los grados de sétimo, octavo y noveno año; y la educación diversificada que la constituye el IV ciclo, y es impartida en planteles denominados liceos, colegios o instituciones diurnas o nocturnas. Al finalizar el III ciclo el estudiantado puede optar por la continuación de sus estudios en la educación diversificada, cuya duración es de 2 a 3 años. También puede seleccionar cualquiera de las siguientes tres ramas: la académica, la artística o la técnica que se diversifica en las siguientes modalidades: industrial, agropecuaria, comercial y servicios. Con este diseño de estructura, todos los caminos quedan abiertos al estudiantado a partir de cualquier ciclo, pues todas las diversificaciones cuentan con un importante núcleo de estudios básicos comunes (Doryan, 1997, p. 2).

En la actualidad, la educación secundaria cuenta con más de 20 modalidades. No obstante, para este estudio se consideran dos:

Modalidad laboratorio
Modalidad académica

Seguidamente se reflexiona sobre el aprendizaje y la evaluación en el proceso educativo en la educación secundaria en la modalidad de liceos académico y laboratorio

\subsection{Aprendizaje y evaluación}

La educación es un hecho y una acción que cobra sentido en el contexto de una sociedad en particular, por ello, se entiende que no es una actividad aislada, ni un proceso técnico que podamos reproducir. La educación es una experiencia y un proceso lleno de vicisitudes, con múltiples aristas que integra situaciones humanas diversas, cotidianas, sociales y personales, que, en los procesos de aula, se visualizan en la práctica pedagógica (Morin, 2005). El proceso educativo involucra los conceptos de enseñanza y aprendizaje, es decir, dos procesos correlativos, inseparables el uno del otro, relacionados de forma interdependiente, aunque reconocemos que muchos de los aprendizajes se obtienen de la vida, sin que medie ningún tipo de enseñanza (Freire, 1993). Se sabe que, dependiendo del contexto, de las estrategias metodológicas aplicadas, la preparación docente, los materiales didácticos utilizados, la actitud, la aptitud, las dificultades socioeconómicas de la población estudiantil, los problemas de aprendizaje y otro tipo de limitaciones, algunas veces esas circunstancias impiden que la enseñanza sea verdaderamente significativa.

La bibliografía señala que el aprendizaje real ocurre en el interior de cada persona que aprende, es subjetivo y su dominio se puede exteriorizar mediante diferentes tipos de evaluaciones (diagnóstica, formativa y sumativa), así como otras acciones que implican mayor creatividad como la elaboración de poesías en diferentes idiomas, obras musicales, piezas teatrales, pinturas, ensayos, proyectos de investigación, entre otras (Vigostky, 1978). En ese sentido, la evaluación desempeña un papel preponderante en los procesos de enseñanza y aprendizaje, ya que le permite al cuerpo docente y a la población estudiantil comprender su evolución en cuanto a su aprendizaje y la producción de conocimientos. Además, en los procesos evaluativos 
cualquier instrumento que se aplique sirve como referente para valorar los aprendizajes construidos y verificar el logro de sus habilidades, destrezas, competencias y actitudes.

\subsection{La evaluación en la educación}

En atención a las normas educativas, y en particular a las de promoción y repitencia, se definen algunos enfoques de evaluación que han evolucionado con el tiempo, según Castillo y Cabrerizo (2003).

- La evaluación como medida. Se centra en dar cuenta de las diferencias individuales entre las personas tomando en cuenta la psicología conductista. No considera como criterio valorar el desarrollo eficiente de los programas.

- La evaluación considerada como el grado de congruencia entre objetivos y su grado de consecución. Esta forma de evaluar considera la evaluación como un proceso sistemático que busca producir cambios en la conducta del estudiantado por medio de procesos de aprendizaje dirigidos al alcance de los objetivos propuestos.

- La evaluación propuesta para la totalidad del ámbito educativo. Esta concepción abarca el rendimiento del alumnado y los factores que intervienen en el proceso educativo: programas, docentes, recursos, contenidos, actividades, metodología, entre otros.

- La evaluación globalizada, formativa, integradora y diferenciada. Esta concepción se compromete con una visión amplia y contextual del proceso educativo que trasciende el centro escolar e involucra las repercusiones socio-históricas, culturales, económicas y políticas en las que está inmerso el estudiantado y sus grupos formadores.

- La evaluación globalizada, formativa, integradora y diferenciada. Esta concepción se compromete con una visión amplia y contextual del proceso educativo que trasciende el centro escolar e involucra las repercusiones socio-históricas, culturales, económicas y políticas en las que está inmerso el estudiantado y sus grupos formadores.

En resumen, en la presente investigación compartimos con Castillo y Cabrerizo (2003) el concepto de evaluación educativa que se fundamenta en un proceso dinámico, abierto y contextualizado, no se trata de acciones puntuales o aisladas, sino que se desarrolla a lo largo de un periodo de tiempo.

En los últimos años, la evaluación se ha convertido en el elemento central de la didáctica; ha prevalecido la concepción técnica, que consiste en la comprobación del logro de los objetivos de parte del estudiantado. En la actualidad, el tema de la evaluación de los aprendizajes es más complejo, ya que intervienen una serie de aspectos tales como los alcances de las políticas educativas, la puesta en práctica de propuestas curriculares, la planificación del proceso educativo, las metodologías aplicadas y los recursos didácticos con que cuenta el centro educativo, la gestión directiva; así como la formación docente y la participación familiar, lo cual contribuye a que la evaluación se considere un requisito esencial para la mejora de la calidad de los procesos de enseñanza y aprendizaje.

Algunos estudios señalan que, en los últimos 20 años, la evaluación educativa ha tenido un importante desarrollo en América Latina, debido a los procesos de reforma en la educación que se llevan a cabo en las diferentes regiones al comprender que el conocimiento y la información producida a través de las investigaciones evaluativas es fundamental para dar cuenta de los logros; conocer los problemas y realizar los cambios necesarios para mejorar los procesos y resultados educativos. Las nuevas reformas tienen un concepto más amplio de calidad en la educación, ya que asumen los aprendizajes cognitivos, valorativos y sociales; que ocurren a lo largo de la vida de manera contextualizada (Murillo y Román, 2010).

Castillo y Cabrerizo (2003) señalan que la evaluación educativa debe facilitar la identidad de la población estudiantil ante el aprendizaje para poder atenderle tal como requiere su situación. Los nuevos enfoques 
de la evaluación se abocan hacia la complejidad de los procesos educativos, por lo que consideran las interrelaciones entre los aprendizajes construidos, la función docente, el currículo, el papel del centro escolar y la participación de la familia, así como la gestión institucional. En ese sentido, promueve una visión contextual que incorpora la dimensión axiológica y socio-histórica que imponen como reto, a la institución escolar, el ofrecer al estudiantado una formación integral para la vida.

Para concluir, si se pretende que la evaluación cumpla con su propósito formativo, debe ofrecérsele al estudiantado la atención pedagógica que requiere, comprendiendo que el aprendizaje que construye desde que ingresa al centro de enseñanza debe ser enriquecido constantemente, de esta manera se podrá asegurar su permanencia en el sistema educativo, por lo que le corresponde al personal docente ajustar su trabajo cotidiano a las necesidades específicas de sus estudiantes, a partir de los lineamientos de la política de promoción y repitencia vigente.

\subsection{Perspectiva teórica conceptual de la investigación}

El propósito general de esta investigación es valorar y otorgar un lugar significativo a la opinión de los actores sociales implicados en la puesta en práctica del sistema de normas regulatorias de la promoción y repitencia, también conocido como el Sistema de Promoción de la Educación Media (SPEM). En este sentido se valora la importancia de esas opiniones, incluyendo las experiencias desarrolladas en las dos instituciones educativas, las cuales nos permiten tener un criterio valorativo para contribuir a solventar sus necesidades respecto a la organización administrativa y curricular, así como llevar a la práctica las lecciones aprendidas del proceso; sin obviar las sugerencias que puedan brindarse a las autoridades educativas.

La puesta en práctica de esta Reforma incide en el cambio educativo, y repercute en todos los ámbitos de la acción pedagógica, especialmente en las relaciones docente-estudiante, centro escolar-estudiante-familia, docente-gestión administrativa, docente-políticas, al considerar que esas relaciones son fundamentales para que cualquier propuesta curricular resulte significativa para el sistema.

Merece especial atención la figura docente por su papel protagónico en el desarrollo y la evaluación de los procesos de aprendizaje, su mediación y los vínculos socio-afectivos que se establecen con la población estudiantil, por lo que su opinión debe ser considerada sin menoscabar su experticia:

Ese cambio educativo constituye un desafío para la población docente, dada su responsabilidad en la formación de estudiantes como ciudadanos críticos y activos; sin embargo, el papel que se les asigna corresponde cada vez más con el de ejecutores del aprendizaje, devaluando su trabajo intelectual y crítico. De esta manera Giroux reivindica al profesor como intelectual transformativo que combina la reflexión con la práctica educativa. (Sarramona, 2000, p. 161)

La evaluación no puede separarse del proceso de construcción del conocimiento, de los criterios para hacer juicio sobre la calidad del trabajo y el compromiso de los grupos en la producción de sus aprendizajes. En todo momento, los criterios para juzgar la calidad del aprendizaje y la funcionalidad del currículo se refieren al grado de autonomía e igualdad experimentado por los miembros del grupo de aprendizaje. Por lo tanto, la perspectiva teórica de las investigadoras se sustenta en la "pedagogía crítica" en calidad de ser una teoría educativa que se centra en el cambio y en la participación de los actores sociales involucrados: directoras, personal de orientación y docentes, estudiantes y familias.

Para concluir, el currículo socio crítico explica los fenómenos educativos como una realidad ideológica socialmente construida, determinada y comprometida con los valores. La relación conocimiento y práctica es dialéctica, por lo que la participación activa es la forma en que se produce la construcción de significados del proceso educativo. Los supuestos descritos permiten ubicar la pedagogía crítica en el contexto de la investigación y en el abordaje teórico, metodológico y axiológico que se asumen en este estudio y se concretan en su planificación. 


\section{4- Metodología}

Para analizar la opinión de la comunidad educativa sobre la "Reforma de las Normas Reguladoras de la Promoción y Repitencia del Sistema Educativo Costarricense", se realiza una investigación de tipo exploratoria en la que se utiliza el método descriptivo mediante técnicas cuantitativas y cualitativas.

El estudio se organiza en etapas:

\section{Etapa: Elaboración y antecedentes del estudio}

Revisión documental emitida por el Ministerio de Educación Pública y el Consejo Superior de Educación sobre los antecedentes de la Reforma.

\section{Etapa: Elaboración teórica*}

Se realiza una revisión bibliográfica sobre la educación secundaria, los procesos de enseñanza y aprendizaje, la evaluación educativa, entre otros temas. Esta segunda etapa se construye de manera constante y tomando en cuenta las revisiones bibliográficas que se consultan para el logro de los objetivos.

\section{Etapa: Elaboración y validación de instrumentos}

La información se recolecta mediante cuestionarios, guías de entrevistas y grupo focal.

Con base en la revisión bibliográfica y en los objetivos del estudio se construyen instrumentos para los directores y directoras de instituciones de educación secundaria, personal de orientación y docente, estudiantes que se encuentran dentro del sistema de promoción, y sus respectivas familias. Asimismo, se elaboran guías de entrevistas para las participantes de las universidades y autoridades del MEP, así como para el grupo focal integrado por docentes que se han agremiado a la Asociación Nacional de Educadores -ANDE-. Los instrumentos indagan el criterio de las personas participantes respecto a las fortalezas y debilidades de la Reforma Educativa, para lograr la promoción y evitar la repitencia en el estudiantado, así como las consecuencias en el rendimiento académico, horarios, permanencia estudiantil, número de estudiantes por grupo, procesos educativos en el salón de clase, carga laboral docente, es decir, en las implicaciones que tiene la Reforma en la organización administrativa y curricular del centro educativo.

Para validar los instrumentos se utilizó la técnica de juicio de personas expertas para lo cual se contó con la participación de una docente universitaria con especialidad en evaluación educativa, que además ejerce funciones en un colegio de educación media, un director y una docente del Departamento de Educación Secundaria de la Escuela de Formación Docente, UCR, quienes revisan los cuestionarios y ofrecen observaciones que se incluyen como parte de los documentos.

\section{Etapa. Negociación de entrada}

Para llevar a cabo el trabajo de campo se negocia la entrada con las autoridades de las direcciones regionales y con las dos instituciones educativas.

\section{Etapa. Selección de la población participante}

Participantes en el estudio son los siguientes:

1. Instituciones educativas: estudiantes a quienes la institución les aplica las normas de promoción, así como a sus familias; también forman parte otras personas involucradas en el sistema: docentes, orientadoras y directoras de las instituciones de educación secundaria seleccionadas. Participan: un liceo académico público (Institución A) y un liceo laboratorio (Institución B) (Ver Tabla 1). Cabe señalar que quienes participan ofrecen su anuencia por medio de dos documentos que solicita la Vicerrectoría de Investigación como parte de las políticas: uno se denomina "consentimiento" y el otro, "asentimiento informado", ambos fueron presentados a la población participante, una vez que se contó con el permiso de la institución. 
TABLA 1

Participantes por institución educativa

\begin{tabular}{lccccc}
\hline $\begin{array}{c}\text { Centro } \\
\text { educativo }\end{array}$ & Directora & Orientadoras & Docentes & Estudiantes & Familias \\
\hline Liceo académico & 1 & 2 & 13 & 30 & 7 \\
\hline Liceo laboratorio & 1 & 2 & 6 & 19 & 3 \\
\hline Total & 2 & 4 & 19 & 49 & 10 \\
\hline
\end{tabular}

1. Autoridades del MEP (tres)

3. Investigadoras universitarias con estudio y experiencia en esta temática (dos)

4. Docentes que forman parte de la ANDE (cuatro)

5. Docentes de universidad que conforman el grupo focal (cuatro)

\section{Etapa. Aplicación de instrumentos}

Se aplica un cuestionario a las directoras de los centros educativos, orientadoras, docentes, estudiantes y familias de los centros educativos participantes.

El procedimiento para aplicar los instrumentos en los centros educativos es el siguiente:

- Se solicita a las direcciones que programen uno o dos días para la aplicación de los cuestionarios.

- Se aplican por grupo: docentes, estudiantes y familias. Para llevar a cabo esta tarea, las directoras convocan a cada grupo a diferentes horas. Se les explica el propósito de nuestra visita, se comentan los objetivos del estudio, su importancia y se aclaran dudas. Se les lee el consentimiento y el asentimiento informados y, posteriormente, lo firman. Se les explica el cuestionario y cada participante lo completa de manera independiente. Dos de las investigadoras estuvieron presentes durante este proceso.

El procedimiento que se sigue para solicitar la información a las autoridades del MEP, a las dos docentes universitarias con estudio y experiencia en la temática, a las académicas universitarias que conforman el grupo focal, y a personal docente que forman parte de la ANDE, es el siguiente:

- Se contacta a cada una de las personas que participarían en la entrevista para solicitar una cita. Se fija la fecha y el lugar de la entrevista.

- Para la organización del grupo focal con docentes de universidad, se conversa con cada persona y se establece un día para llevar a cabo esta tarea.

En todos los casos se les explica el propósito de la entrevista, se comentan los objetivos del estudio y su importancia. Se les entrega el consentimiento y asentimiento informados para su respectiva firma. Luego se les expone la guía de entrevista y se inicia el trabajo; se graba la conversación, previa autorización. Las tres investigadoras estuvieron presentes durante este proceso.

\section{Etapa. Análisis de la información}

Con el propósito de conocer el impacto del Sistema de Promoción de la Educación Media (SPEM) a nivel nacional se lleva a cabo el siguiente procedimiento:

- Se analizan los datos que posee el Ministerio de Educación Pública respecto a la repitencia, reprobación y deserción del estudiantado, que se encuentra en el III ciclo de la EGB y el ciclo diversificado durante los años 2000 al 2011. 
- La información emitida por cada institución educativa participante se analiza de manera individual, debido a que corresponde a modalidades de educación secundaria diferentes. Lo anterior permite contextualizar cada centro educativo, así como caracterizar al personal docente y a la población estudiantil que participan en la investigación.

- Los datos recolectados mediante los cuestionarios aplicados a docentes y estudiantes se analizan de forma cuantitativa y cualitativa de acuerdo con la información suministrada, y como criterio de credibilidad se utiliza la triangulación de fuentes.

Con base en los objetivos del estudio y el análisis de la información recolectada se definen las siguientes categorías y subcategorías de análisis:

- Educación secundaria en cifras

- Criterio de la comunidad educativa respecto a los siguientes aspectos:

Organización administrativa de la institución para implementar el SPEM: Inducción. Horarios. Carga laboral. Espacio físico

Organización curricular: Planeamiento. Evaluación de los aprendizajes. Atención a estudiantes

Percepción de las consecuencias para el estudiantado

Valoración de la experiencia

Recomendaciones para mejorar el SPEM

\section{Etapa. Elaboración de conclusiones y recomendaciones}

Las conclusiones y recomendaciones surgen de dos fuentes:

- Se toman en cuenta los resultados de los cuestionarios y entrevistas en los que participan los diferentes actores sociales: cuerpo administrativo, docentes, estudiantes y familias, así como las opiniones externadas por las autoridades del MEP, de las docentes académicas, las opiniones generadas por el grupo focal y por participantes de la ANDE.

- En esta etapa también se involucran los criterios de las investigadoras, producto de sus experiencias como docentes en el sistema educativo nacional, de su labor investigativa y de su trabajo como supervisoras en instituciones de educación secundaria.

\section{Etapa. Divulgación de los resultados del estudio}

- Como parte de la divulgación, se elabora el presente artículo científico para ser publicado en una revista reconocida internacionalmente.

- Los resultados de esta investigación se presentan al Consejo Superior de Educación a inicios del año 2015, posteriormente este Consejo crea una comisión para revisar los efectos de la Reforma, en la que participan las autoras del presente estudio.

\section{ANÁLISIS DE RESULTADOS}

El análisis se construye a partir de los objetivos de la investigación, de los textos indagados como parte de la bibliografía, de los documentos emanados por el MEP sobre la Reforma de las normas reguladoras de la promoción y repitencia dentro del Sistema Educativo Público Costarricense y por los aportes de los actores 
educativos que participan en este estudio: comunidad educativa de las Instituciones A y B, autoridades del MEP, personal académico universitario, docentes de ANDE.

En primera instancia se presentan consideraciones que se relacionan con los datos estadísticos del MEP sobre exclusión, repitencia, aprobación y reprobación de la educación secundaria del año 2000 al 2012. Luego se dan a conocer las siguientes categorías de análisis, las cuales responden a los objetivos del estudio.

\section{Educación secundaria en cifras: exclusión, repitencia, aprobación y matrícula}

De acuerdo con los datos emanados por el Departamento de Estadística del MEP, la Reforma de las normas reguladoras de la promoción y repitencia dentro del Sistema Educativo Público Costarricense presenta el siguiente objetivo educativo:

...se trata de definir los criterios de evaluación, promoción y repitencia que mejor contribuyan para alcanzar los objetivos de aprendizaje para todas y todos los estudiantes. Por este motivo, en el 2008, se impulsó una reforma a estas normas, buscando atacar algunas de las que consideramos causas artificiales del fracaso escolar, y de una repitencia injustificada, innecesaria y de severas consecuencias, en términos de la deserción y la baja cobertura de nuestra educación secundaria. (MEP, 2011, p. 4)

De esta manera, la implementación de la Reforma en cuestión le va a permitir a la población estudiantil de la educación secundaria, la oportunidad de repetir únicamente las materias que reprueba y no todas, así como adelantar materias del siguiente año, lo que contribuiría a que un mayor número de estudiantes se pueda mantener dentro del sistema educativo, y pueda concluir con la obtención del Bachillerato. Este objetivo es muy bien valorado por la totalidad de las personas que participan en el estudio. Sin embargo, aunque los porcentajes de exclusión han bajado, aún no se presenta una tendencia sostenida, es así como en el año 2000 la exclusión de la población estudiantil de III ciclo y educación diversificada de colegios académicos diurnos y técnicos diurnos fue de un $13.3 \%$, en el 2008 baja a un $11.8 \%$, en el 2009 año que se aplica la Reforma, se reduce a un $10.7 \%$, en el 2010 baja a un $9.7 \%$, en el 2011 sube a un $11 \%$ y en el 2012 llega un $9.6 \%$.

En cuanto a la repitencia, a partir de la puesta en práctica del Sistema de Promoción de la Educación Media (SPEM), se han presentado altibajos en esos porcentajes, ya que desde el 2011 la tendencia es a la baja, pues en ese año fue de un 14.3\%, mientras que en el 2013 del 10.9\%, dato que llama la atención debido a que podría pensarse que con el SPEM aumentaría el número de repitentes debido a que el estudiantado que "arrastra" materias se ubicaría dentro del grupo de repitentes; sin embargo, esa situación no se evidencia en los datos del MEP.

La repitencia se asocia a un bajo rendimiento académico de acuerdo con las pruebas internacionales que realiza la UNESCO (2012) y la OCDE en diversos países. Es conocido que la repitencia es una de las causas del abandono escolar, lo que conlleva a consecuencias negativas para la persona en cuanto a su ingreso al mercado laboral, y a su calidad de vida futura.

En relación con los porcentajes de aprobación, estos no han aumentado de manera sostenida después de la implementación de la Reforma, ni tampoco han disminuido los porcentajes de reprobación de forma constante. Es más, en el 2000 se tenían mayores niveles de aprobación -80.9\%- y menores de reprobación $-19.1 \%$-, en relación con los obtenidos en el $2011,-78.2 \%$ y $21.8 \%$ - respectivamente.

En cuanto a las tasas netas de matrícula de la educación secundaria, el Informe del Estado de la Educación indica que del 2006 al 2012 "solo creció seis puntos porcentuales, en contraste con los trece puntos registrados en el periodo 2000-2005. A este ritmo el país se encuentra lejos de alcanzar la universalidad en el corto o mediano plazo" (CONARE, 2013, p. 143).

Lo anterior evidencia que la aplicación de los lineamientos de la Reforma aún no han producido los cambios cuantitativos esperados, puesto que la repitencia y la exclusión estudiantil del sistema educativo es un problema multifactorial en la que intervienen numerosas variables, por lo que esta situación se debe resolver de manera integral y con la participación del MEP y de otros actores sociales. 


\section{Criterio de la comunidad educativa sobre la Reforma}

Las reflexiones que se presentan integran el criterio del personal administrativo, orientadoras, docentes, estudiantes, familias de los centros educativos A y B que forman parte del estudio, así como de las personas especialistas que participan en la investigación, respecto a las categorías y subcategorías. En términos generales, esas personas consideran pertinente que el estudiantado únicamente repita o "arrastre" las materias que reprobó y no todas, un grupo de familias prefiere que sus hijas e hijos repitan todo el año, por lo que la mayoría no está de acuerdo con esta Reforma; pues les preocupa su organización administrativa. En cuanto a la organización de los horarios, el tiempo libre del estudiantado en la institución, la falta de espacio en los centros educativos, la cantidad de grupos numerosos; y el énfasis que el MEP le brinda al aspecto cuantitativo, respecto a bajar los porcentajes de exclusión más que al mejoramiento de la calidad de la educación y a la cantidad de años que el estudiantado debe permanecer en la institución para aprobar un nivel de educación secundaria, es una situación que debe resolver lo antes posible el MEP.

\section{Organización administrativa para implementar la Reforma}

Las instituciones educativas participantes en el estudio indican que la organización administrativa del SPEM es coordinada por la dirección y en ella participa el Departamento de Orientación, ente encargado de elaborar los horarios de manera conjunta con estudiantes, con el propósito de brindarles apoyo, analizar los resultados que van obteniendo, así como dar seguimiento a las adecuaciones curriculares y a otras situaciones especiales que surgen durante el proceso. Cabe señalar que esta labor se coordina con el personal docente. Además, la institución se mantiene en contacto con las familias, así como con los profesores y profesoras guías de cada grupo; en esta tarea, también colabora el Comité de Evaluación del Colegio. En la Institución B, por ser modalidad laboratorio, funciona un Comité que coordina la implementación de proyectos de recuperación para que el estudiantado tenga la oportunidad de ganar la materia perdida; además, cuenta con una coordinadora académica que organiza los proyectos con estudiantes que "arrastran" materias para hacer uso del tiempo libre de manera efectiva.

Seguidamente se hace un comentario con base en el criterio de las personas que participaron en el estudio sobre algunos aspectos relacionados con la puesta en práctica del SPEM.

\section{- Inducción}

Según las respuestas emitidas por las personas que participan, la puesta en práctica de la Reforma ha traído una gran cantidad de problemas administrativos que le preocupa a la comunidad educativa, considera que se implantó de manera precipitada, sin una consulta formal al personal docente ni a los gremios, con poca inducción, no se brindó un proceso de sensibilización, ni de capacitación para implementarla. Esa realidad produjo, en parte del personal docente y administrativo de las instituciones, un rechazo a la Reforma, al sentirse excluido como parte del proceso, esta situación debió tomarse en cuenta por parte de las autoridades del MEP, para asegurar el éxito deseado hacia la puesta en práctica de una propuesta que pretendía introducir cambios en beneficio de la educación secundaria. Una cantidad importante de participantes indica que la Reforma debió experimentarse, en la modalidad de "plan piloto", con el propósito de valorar sus fortalezas y limitaciones antes de implementarla a todo el país. En términos generales, los estudiantes y las familias indican que la información brindada por las instituciones educativas A y B sobre el SPEM no fue compresible ni suficiente. 


\section{- Horarios}

La propuesta definitiva del horario para la población estudiantil que pertenece al SPEM representa una gran preocupación, ya que en algunas instituciones transcurre un mes o más tiempo para que el estudiantado conozca su horario, lo que implica una importante cantidad de adolescentes sin ocupaciones determinadas durante ese lapso. En el caso de la Institución A que corresponde a una Dirección 3, con una matrícula de 1400 estudiantes, de los cuales 251 "arrastran" materias, se indica que deben esperar aproximadamente un mes para organizar los horarios de estos estudiantes, mientras que en la Institución $\mathrm{B}$, que corresponde a una Dirección 1 con una matrícula de 451 estudiantes, de los cuales 30 arrastran, tardan quince días en este proceso; es importante mencionar que esta institución pone en práctica el Programa Informático de Alto Desempeño (PIAD) para elaborar los horarios de la institución, lo que facilita esta fundamental tarea.

Con respecto a los horarios, el personal docente manifiesta que la población estudiantil tiene mucho tiempo libre en la institución, lo que facilita que deambulen por los corredores y, en consecuencia, distraen a quienes se encuentran en lecciones. Esta situación facilita la formación de pandillas y favorece la indisciplina y el desorden en la institución. El estudiantado manifiesta que durante ese tiempo hace lo "que más le convenga", otros sectores informan que realizan tareas o estudian. A algunas de las familias les preocupa que no saben lo que hacen sus hijos e hijas durante el tiempo que tienen libre, otras señalan que conocen las actividades que realizan sus hijos e hijas: "caminar por los pasillos", "escaparse de las lecciones" o "irse para la casa a jugar".

Las directoras de ambas instituciones consideran la importancia de ofrecer clubes o actividades extracurriculares al estudiantado para que aproveche el tiempo libre, pero la falta de espacio y de recursos limita poner en práctica esta estrategia.

\section{- Carga laboral}

De acuerdo con el criterio del personal docente y administrativo de esas instituciones educativas, y de los sujetos especialistas consultados, el SPEM implica más trabajo debido a que se deben elaborar horarios personalizados, dar seguimiento de forma individual y grupal al estudiantado y a las familias, atender grupos numerosos y, en algunos casos, aumentar el número de personas que vigilen la institución; además, para las directoras de ambas instituciones, es necesario contar con más personal docente y administrativo que organice actividades extracurriculares o planes de recuperación para atender de una manera mas efectiva e individual a quienes "arrastran" materias.

\section{- Espacio fisico}

El espacio físico es otro de los aspectos que más preocupa a quienes participaron en esta investigación, debido a que la carencia de espacio no favorece una adecuada atención del alumnado, en algunos casos los grupos son demasiado numerosos, y al no contar con suficientes pupitres o mesas, deben recibir las lecciones totalmente hacinados y sentados en el piso de las aulas, lo que no favorece los procesos de enseñanza y aprendizaje, ni una atención personalizada. Por otra parte, la falta de aulas y otros espacios se convierte en una limitación para ofrecer talleres, clubes o tutorías durante el tiempo libre del estudiantado. Lo anterior produce que numerosos estudiantes vaguen por la institución generando desorden, contaminación sónica e indisciplina. 


\section{- Organización curricular del SPEM}

El propósito de esta categoría es conocer si se producen cambios relevantes en el planeamiento, evaluación de los aprendizajes y en la atención a la población estudiantil que realiza el personal docente a partir de la puesta en práctica del SPEM. Los resultados indican que la mayoría de docentes no realizó transformaciones significativas en esos aspectos. Una pequeña parte informa que ofrece lecciones de recuperación o una atención más personalizada a sus estudiantes del SPEM, con necesidades educativas especiales y adecuaciones curriculares, lo cual coincide con lo que manifiesta la población estudiantil al indicar que el apoyo académico que reciben, lo brindan las familias al contratar docentes para que les brinden tutorías, también manifiestan que reciben apoyo de tipo académico de compañeros, compañeras y amistades. No obstante, pocas personas señalan que reciben atención individualizada de parte de sus profesores.

\section{- Percepción de las consecuencias para el estudiantado}

Las personas participantes en el estudio consideran que una consecuencia positiva del SPEM es que el estudiantado no tiene que repetir un año completo, sino únicamente las materias reprobadas; además, cuenta con la opción de adelantar materias del siguiente año. Consideran que ayudan a la población estudiantil con necesidades educativas especiales porque se focalizan en pocas materias y logran mejorar su rendimiento académico; a pesar de esas ventajas, la mayoría no está de acuerdo con esta Reforma.

El personal docente y administrativo de los centros educativos, familias y especialistas que participaron en el estudio señalan como consecuencias negativas del SPEM las siguientes: el estudiantado no aprovecha el tiempo libre a pesar de las opciones que le brinda la institución, lo que provoca violencia, indisciplina y desorden en los centros educativos; la organización administrativa y curricular que demanda este sistema no es conveniente para la etapa de desarrollo en la que se encuentra esta población; algunos grupos son muy numerosos, lo que afecta los procesos de enseñanza y aprendizaje de manera efectiva; con frecuencia se presenta un aumento en las ausencias a las lecciones; tampoco favorece la identificación de este tipo de estudiante con el grupo, las cifras emitidas por el MEP señalan que el alumnado deserta de las materias que adelanta después del receso de medio año, e indican "de todas maneras siempre tenemos que repetir al menos un año”. La Institución A señaló que en el año 2011, ningún estudiante había aprobado las materias que tenía rezagadas. En la Institución B la aprobación de esas materias había sido alrededor del 75\%, cabe recordar que se trata de un liceo laboratorio que tiene características diferentes al resto de instituciones de educación secundaria.

La opinión de la población estudiantil de ambas instituciones está dividida: una parte considera que el SPEM es positivo debido a que le permite repetir únicamente las materias reprobadas y adelantar otras, y además, da más tiempo libre; otra considera que no trae consecuencias positivas, ya que "siempre se pierde un año lectivo y porque los docentes no se encuentran preparados para aplicar de manera eficiente el SPEM". Considera que era mejor el anterior sistema.

\section{- Valoración de la experiencia}

Que el estudiantado repita solo las asignaturas que reprueba con la posibilidad de adelantar asignaturas de niveles superiores

El personal docente y administrativo, las familias de las Instituciones A y B, y las personas especialistas que participaron en la investigación valoran como positivo que el SPEM le dé la oportunidad al estudiantado de repetir únicamente las materias que reprobó y pueda adelantar otras, también consideran que al matricular menos materias le permite mejorar su rendimiento académico. No obstante, en términos 
generales, cuestionan y consideran negativa esta condición, pues mencionan que el SPEM no ha logrado bajar la repitencia ni la deserción en las instituciones participantes, debido a que ambas problemáticas son multicausales.

Se reitera que la organización del SPEM ha producido un desorden administrativo que afecta a estudiantes de la siguiente manera: grupos recargados; estudiantes con mucho tiempo libre deambulando por la institución lo que causa problemas de indisciplina, violencia, consumo de drogas, y que se conviertan en distractores para quienes permanecen atentos en las aulas; también se incrementa el ausentismo durante las lecciones, la desmotivación y la falta de interés por parte de la población adolescente que "arrastra materias". Además, la mayoría de estudiantes no se identifica con ninguno de los grupos a los que debe asistir a recibir las lecciones, y en algunos casos se presentan problemas con estudiantes por su "sobre edad".

Por otra parte, se indica que las instituciones educativas no tienen los recursos humanos suficientes para ofrecer actividades extracurriculares y cursos de recuperación o "centros" a la población estudiantil durante "los cajones o espacios libres".

Consideran que al SPEM lo que le interesa es aumentar los porcentajes de permanencia del estudiantado en el sistema educativo, más que el aprendizaje y el mejoramiento de la calidad de la educación que recibe esta población.

La mayoría de las familias no está de acuerdo con el SPEM, indica que confunde al estudiante y a sus familiares ya que se les ofrece información engañosa, pues el alumnado finalmente siempre debe repetir el año. Argumentan que es un sistema que no toma en cuenta el aspecto socioafectivo del estudiantado, ya que este pierde el sentido de pertenencia a un grupo o peor aún, a una generación, además, preocupa el tiempo libre que tiene y que desaprovecha.

La población estudiantil de las Instituciones A y B señala como aspectos positivos del SPEM los siguientes: tiene lecciones libres, horarios más cortos, adelantan materias, no deben repetir las materias aprobadas y amplían su grupo social. Como aspectos negativos indican que este sistema es un desperdicio de tiempo y promueve la ausencia a clases, no logran sentirse incluidos en los grupos a los que asisten, consideran que los horarios están mal elaborados y se presenta desorden en las lecciones.

Que el curso lectivo se aprueba con el promedio anual de los tres trimestres

Respecto a esta disposición, en la Institución A están de acuerdo, mientras que en la Institución B la posición está dividida.

Que los cursos se aprueben con su nota ponderada anual (el tercer trimestre no aplaza)

La mayoría del profesorado de la Institución A está de acuerdo y en la Institución B las opiniones están divididas.

Que el estudiantado no pierde el curso lectivo por notas de conducta

En la Institución A están divididas las opiniones y en la Institución B la mayoría no está de acuerdo.

Las personas participantes en el estudio consideran que el SPEM es un esfuerzo del MEP para retener a la población estudiantil al sistema educativo, pero no es suficiente para lograrlo; su puesta en práctica ha demostrado que es "un engaño", porque el estudiantado siempre va a tener que cursar uno o dos años más para pasar al siguiente nivel; algunos sujetos piensan que el SPEM debe replantearse o eliminarse, porque no ha traído cambios importantes que beneficien al estudiantado. Se requiere de una atención más personalizada a la población estudiantil con bajo rendimiento académico, sobre todo si pertenece a comunidades en riesgo social, para lo cual se se necesitan más recursos humanos y materiales en las instituciones educativas.

\section{Recomendaciones}

Las recomendaciones que brindan quienes participan en el estudio, van desde eliminar el SPEM hasta reelaborarlo para corregir lo que no funciona, la mayoría de las personas manifiesta no estar de acuerdo con este sistema tal y como funciona en la actualidad. 
Quienes sugieren eliminarlo, y así lo considera la mayoría, externan los siguientes argumentos:

- Volver al modelo anterior, ya que el sistema actual ha demostrado que no contribuye a mejorar el rendimiento académico del estudiantado, ni a bajar de manera significativa la repitencia ni la exclusión en la educación secundaria, además, no se enfoca en mejorar la calidad de la educación que recibe la población estudiantil, sino en bajar los porcentajes de exclusión y repitencia. Consideran que repetir un año completo, como se hacía en el modelo anterior, contribuye a lograr una mejor formación integral en el alumnado. Asimismo, formulan las siguientes propuestas para mejorar el actual sistema:

- Ofrecer al estudiantado diversas oportunidades durante el año, para que elabore trabajos especiales y pueda realizar exámenes o evaluaciones diversas, con el fin de que demuestre el manejo de los contenidos que no domina. Esto se podría realizar al concluir cada trimestre, durante el receso de medio año, pero no al concluir el curso lectivo.

- Brindar durante los recesos del curso lectivo o en el periodo del "Plan 200 que organiza el MEP” (dos semanas al finalizar el año), tutorías y cursos de verano para estudiar o fortalecer los contenidos que no se dominan, y de esta manera aprobar las materias perdidas.

- Aumentar el número de convocatorias a estudiantes aplazados.

- Que el Departamento de Orientación y el profesorado (en general), brinden mayor seguimiento a estudiantes con un rendimiento académico deficiente, ofreciéndoles tutorías, así como solicitando a docentes jubilados y otras personas interesadas en los procesos educativos de la comunidad, su colaboración para que ofrezcan apoyo académico; también se le puede pedir ayuda por medio de la planificación de lecciones de recuperación a estudiantes de la universidad que cursen los niveles avanzados de la misma institución. Se sabe que siempre hay personas que ofrecen de manera desinteresada sus servicios.

- Crear redes de apoyo para estudiantes con rendimiento académico deficiente, las que podrían ser coordinadas por el profesorado guía y con la participación de estudiantes, familias y Departamento de Orientación de la Institución.

- Plantear la promoción por ciclo y no por año. Es decir, que el estudiantado pase de $7^{\circ}$ a $8^{\circ}$ y luego a $9^{\circ}$, durante este tiempo podría ir realizando diferentes pruebas o trabajos especiales respecto a los contenidos que no domina, a la vez que avanza hasta $9^{\circ}$. De igual forma con el ciclo diversificado.

Los sujetos participantes que sugieren hacerle ajustes al SPEM para mejorarlo señalan lo siguiente:

- Que el MEP ofrezca capacitación al personal docente y administrativo sobre lineamientos específicos del SPEM, pues la experiencia señala que gran parte del personal docente y administrativo desconoce aún cómo hacer realidad la filosofía de este sistema para colaborar con una población estudiantil que posee una gran cantidad de carencias.

- Contar con más recursos humanos y materiales para mejorar la organización del SPEM y brindar a la población estudiantil seguimientos personalizados, tutorías, actividades extracurriculares y clubs durante el tiempo que tienen libre en la institución.

- Que las instituciones educativas realicen un estudio de las materias reprobadas con mayor frecuencia, y solicitar al MEP la aprobación de uno o dos grupos más de esas materias para el siguiente curso lectivo.

- Organizar más grupos de apoyo en la institución educativa para el seguimiento del estudiantado que "arrastra" materias con el fin de darle una atención individualizada que le permita mejorar su rendimiento académico.

- El profesorado debe conocer realmente al alumnado, sus capacidades y limitaciones, por lo que se sugiere que el área administrativa organice sus horarios de manera que el mismo docente pueda 
trabajar (de ser posible) de manera continua con el grupo de estudiantes durante $7^{\circ}, 8^{\circ}$ y $9^{\circ}$, y lo mismo en Educación Diversificada.

- Brindar a las familias más información sobre el SPEM y sobre el rendimiento académico del estudiantado.

- Crear diversos programas que comprometan a las familias.

- Organizar de una manera más adecuada los horarios del estudiantado que repite materias.

- Realizar reuniones periódicas con la población estudiantil que arrastra materias, de esta manera, se podrá dar mayor seguimiento con respecto a su avance académico.

- Organizar grupos integrados por estudiantes que repiten por nivel y por materia.

- Que la población estudiantil solo lleve únicamente las materias que perdió y no adelante otras.

- Variar el periodo de tiempo para aprobar las materias perdidas, no esperar un año completo para hacerlo.

- Ofrecer programas de recuperación vespertinos.

Asimismo, las personas que participaron en la investigación consideran la importancia de:

- Evaluar los resultados de la Reforma de manera integral, tomando en cuenta las consecuencias positivas y negativas del actual rendimiento académico de la población estudiantil que se encuentra en el SPEM, además, es necesario conocer si el estudiantado que "arrastra" materias logra aprobarlas en 1 o 2 años, así como saber, si las materias que adelanta, las aprueba o las reprueba, también es esencial conocer cuánta cantidad de estudiantes logra aprobar las materias que arrastra y las que adelanta para conocer el impacto del SPEM en la actualidad.

- Mejorar la formación de docentes, es necesario que el profesorado tenga un mayor conocimiento sobre: la adolescencia, pedagogía, manejo de grupos, técnicas de trabajo en grupo, dominio de contenidos; que participen en prácticas supervisadas más amplias, profundas y reflexivas en contextos escolares diversos.

- Analizar el currículo de la educación secundaria con el fin de que sus contenidos sean pertinentes y relevantes.

- Realizar pruebas de conocimiento y aptitud para el ingreso al trabajo docente por parte del MEP, tanto al personal docente como administrativo. Dar especial atención a la contratación de directoras y directores por la importancia que tiene el liderazgo académico en la transformación y mejoramiento de las instituciones educativas y, por ende, en el rendimiento escolar de la población estudiantil.

- Cambiar el sistema de contratación del personal docente de educación secundaria en el MEP, para que se contrate por tiempo y no por lección.

\section{REFLEXIÓN FINAL}

Se reconoce que la situación de la educación secundaria en nuestro país es crítica; según el IV Informe del Estado de la Educación, únicamente dos terceras partes de jóvenes entre 12 y 16 años se encuentran matriculados (CONARE, 2013), también sabemos que el MEP ha puesto en marcha numerosos programas con el propósito de mantener a la población estudiantil en el sistema educativo, y que el SPEM se considera un esfuerzo que hace el MEP para disminuir la exclusión del estudiantado. Sin embargo, los resultados de este estudio evidencian que aún quedan muchos retos y desafíos que como ciudadanas y ciudadanos debemos asumir; es urgente revisar el modelo de la educación media que se ofrece en la actualidad, debido a que no está cumpliendo con las expectativas de la comunidad nacional e internacional, ni con las características, necesidades e intereses de la población estudiantil, así mismo se requiere analizar los modelos de formación docente que se desarrollan en nuestro país y en otras latitudes, con el propósito de mejorar los procesos de formación de profesionales en educación para hacer frente a las demandas con miras al desarrollo integral 
del estudiantado y a promover la educación a lo largo de la vida. Desde este punto de vista es inaplazable transformar el sistema de contratación de los servicios docentes por parte del Estado para que seleccione al personal docente con criterios de idoneidad, más allá de la obtención de un título universitario o de la acreditación de una carrera. Urge que reconozca sus aptitudes, actitudes, conocimientos, destrezas de pensamiento, habilidades sociales, compromiso y su buena disposición para ejercer la profesión docente.

Es perentorio el rol que deben asumir las directoras y directores de las instituciones educativas del país, cargo que exige la función gestora académica y administrativa de los procesos educativos que se llevan a cabo en la institución, por lo que tanto, el proceso de elección para ocupar estos puestos debe ser exhaustivo para que las personas elegidas cuenten con las competencias profesionales y actitudinales que les permitan desempeñar, de manera óptima, su función y mejorar los servicios que brinda la institución a la comunidad educativa.

Otro actor social indiscutible es la familia, la cual debe asumir su rol formador como primer agente educativo, así como su compromiso con el seguimiento y acompañamiento de sus hijas e hijos durante su proceso de formación escolar, en comunicación con la institución educativa para propiciar el éxito académico del estudiantado.

De igual forma, el Estado, debería brindar oportunidades de formación a las familias para cumplir con esta función social, lo que contribuiría a disminuir la exclusión del estudiantado del sistema educativo.

Si deseamos mejorar el servicio educativo que recibe la población estudiantil de la educación secundaria, debemos comprender el compromiso que tiene nuestro país por brindarle, no solo el derecho a una educación de calidad, sino a una educación que le permita desarrollar sus aptitudes críticas, habilidades creativas, sociales, para la convivencia, el respeto a la diversidad, al desarrollo sostenible y a la vida democrática. Lo anterior requiere de la aplicación de estrategias pedagógicas innovadoras y adecuadas a sus condiciones personales. Los criterios señalados y otros que puedan surgir con el desarrollo de investigaciones para favorecer la permanencia del estudiantado en el sistema educativo, para lograr la conclusión de la Educación Diversificada y obtener el grado de Bachillerato en la Enseñanza Secundaria, se consideran criterios que favorecen la posibilidad de ingresar a la educación superior, y de esta manera, fortalecer sus opciones de empleo y su calidad de vida.

\section{ReFERENCIAS}

Castillo, S. y Cabrerizo, J. (2003). Evaluación educativa y promoción escolar. Madrid: Pearson Educación.

Consejo Nacional de Rectores (CONARE). (2008). II Estado de la Educación. San José, Costa Rica: Programa Estado de la Nación y Desarrollo Sostenible. San José, Costa Rica. Consejo Nacional de Rectores.

CONARE. (2013). IV Estado de la Educación. San José. Costa.Rica.: Programa Estado de la Nación y Desarrollo Sostenible. Consejo Nacional de Rectores

Consejo Superior de Educación. (2008). Actas ordinarias N. . 50-2008, N. ${ }^{\circ}$ 51-2008, N.o 53- 2008. San José, Costa Rica: Autor.

Doryan, E. (1997). Sistema Educativo Nacional de Costa Rica. MEP. Recuperado de http://www.oei.org.co/quipu/ costrica/index.html

Freire, P. (1993). Pedagogía de la Esperanza. México: Siglo XXI.

Ministerio de Educación Pública (MEP). (1971). Plan Nacional de Desarrollo Educativo. San José, Costa Rica: Ministerio de educación Pública.

MEP. (2008). Reforma integral de las normas reguladoras de la promoción y repitencia dentro del sistema educativo público costarricense. San José, Costa Rica: Ministerio de educación Pública.

MEP. (2011). Normas reguladoras de la promoción y repitencia dentro del sistema educativo público costarricense. San José, Costa Rica: Ministerio de Educación Pública. 
Morin, E. (2005). Epistemología de la complejidad. En Fried Schnitman (Comp.), Nuevos paradigmas, cultura y subjetividad. (pp. 421-442). Buenos Aires: Paidós.

Murillo, J. y Román, M. (2010). Retos en la evaluación de la calidad de la educación en América Latina. Revista Iberoamericana de Educación, 53, 97-112.

Sarramona, J. (2000). Teoría de la educación. Reflexión y normativa pedagógica. Barcelona: Editorial Ariel.

UNESCO. (2012). Global Education DIGEST 2012. Instituto de Estadística de la UNESCO. Recuperado de http:// www.uis.unesco.org/Education/Pages/ged-2012-press-release.aspx

Vigostky, L. (1978). Pensamiento y lenguaje. Buenos Aires: La Pleyade.

\section{BY-NC-ND}

